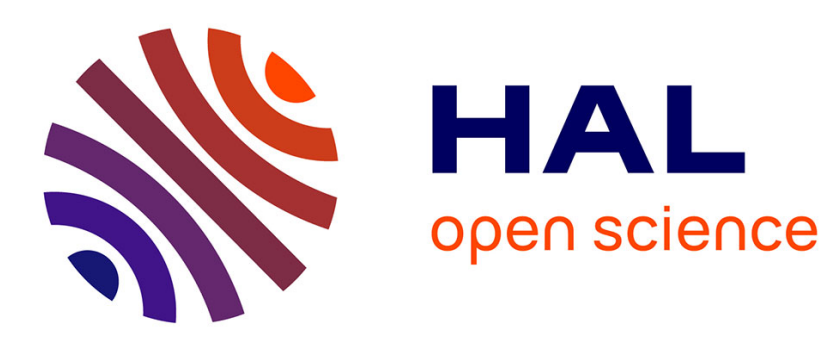

\title{
Une forme dangereuse d'érosion : les chutes de pierres dans la basse vallée de la Vésubie (Alpes-Maritimes) \\ Pierre Carrega
}

\section{To cite this version:}

Pierre Carrega. Une forme dangereuse d'érosion: les chutes de pierres dans la basse vallée de la Vésubie (Alpes-Maritimes). Méditerranée : revue géographique des pays méditerranéens, 1983, 49 (3), pp.53-60. 10.3406/medit.1983.2135 . hal-02298719

\section{HAL Id: hal-02298719 https://hal.science/hal-02298719}

Submitted on 27 Sep 2019

HAL is a multi-disciplinary open access archive for the deposit and dissemination of scientific research documents, whether they are published or not. The documents may come from teaching and research institutions in France or abroad, or from public or private research centers.
L'archive ouverte pluridisciplinaire HAL, est destinée au dépôt et à la diffusion de documents scientifiques de niveau recherche, publiés ou non, émanant des établissements d'enseignement et de recherche français ou étrangers, des laboratoires publics ou privés. 


\title{
Une forme dangereuse d'érosion : les chutes de pierres dans la basse vallée de la Vésubie (Alpes-Maritimes)
}

Pierre Carrega

\begin{abstract}
In Alpes Maritimes, near Nice, the falls of lump threaten frequently many roads. Studied along a section of the Vesubie Valley, the fallen amounts are connected to two sorts of explanation : the variables of the climate (rain, frost) and the variables of the topography and geological structure (height of the bank, slope of geological layers, fissures ...) . The part of the seasons, varying according to the spots, is shown up.
\end{abstract}

\section{Résumé}

Dans l'arrière pays niçois, les chutes de blocs menacent fréquemment de nombreuses routes. Etudiées sur un tronçon de la vallée de la Vésubie, les quantités éboulées sont reliées à deux familles d'explication : les variables climatiques (pluie, gel) et les variables topo-structurales (hauteur du talus, pendage, fissures etc..) . Le rôle des saisons, variant selon les endroits, est mis en évidence.

\section{Citer ce document / Cite this document :}

Carrega Pierre. Une forme dangereuse d'érosion : les chutes de pierres dans la basse vallée de la Vésubie (Alpes-Maritimes). In: Méditerranée, troisième série, tome 49, 3-1983. pp. 53-60;

doi : https://doi.org/10.3406/medit.1983.2135

https://www.persee.fr/doc/medit_0025-8296_1983_num_49_3_2135

Fichier pdf généré le 25/04/2018 


\title{
Une forme dangereuse d'érosion : les chutes de pierres dans la basse vallée de la Vésubie (Alpes-Maritimes)
}

P. CARREGA*

Résumé - Dans l'arrière pays niçois, les chutes de blocs menacent fréquemment de nombreuses routes. Etudiées sur un tronçon de la vallée de la Vésubie, les quantités éboulées sont reliées à deux familles d'explication : les variables climatiques (pluie, gel) et les variables topo-structurales (hauteur du talus, pendage, fissures etc...) . Le rôle des saisons, variant selon les endroits, est mis en évidence.

\begin{abstract}
In Alpes Maritimes, near Nice, the falls of lump threaten frequently many roads. Studied along a section of the Vesubie Valley, the fallen amounts are connected to two sorts of explanation: the variables of the climate (rain, frost) and the variables of the topography and geological structure (height of the bank, slope of geological layers, fissures ...). The part of the seasons, varying according to the spots, is shown up.
\end{abstract}

Malgré le haut degré de technologie et de développement atteint par les pays industriels, ceux-ci ne sont pas à l'abri des paroxysmes de la nature à tel point qu'en France, par exemple, la conscience de ces dangers a récemment abouti à la création d'un «Haut Commissariat à la prévention des Risques Naturels».

Les risques naturels ne sont pas seulement d'ordre sismique ; en terme de probabilité et de fréquence, ils concernent bien davantage les phénomènes météorologiques et leurs conséquences induites, qu'elles soient d'ordre hydrologique ou géomorphologique : chutes de neige, inondations, sécheresse, glissement de terrains, éboulements,etc...

Dans les Alpes Maritimes, les chutes de blocs et de rochers, sont l'un des fléaux naturels majeurs car ils menacent parfois certaines constructions mal implantées et gênent souvent considérablement la circulation automobile, et ce, d'autant plus qu'aujourd'hui les axes routiers majeurs desservant l'arrière-pays empruntent les fonds de vallées, contrairement aux coutumes antérieures qui leur faisaient préférer les sommets de collines et les crêtes.

Ainsi, tout véhicule circulant au fond d'une vallée comme celle de la Vésubie par exemple encourt un certain risque de heurter des blocs déjà éboulés sur la chaussée au détour d'un rivage, ou pire, d'être écrasé par ces blocs en cours d'éboulement.

Ce risque évolue cependant en fonction de deux variables majeures : le temps et l'espace. Le but de cette étude est justement d'interpréter les résultats d'une campagne de mesures et d'en extirper des lois prévisionnelles à partir de la recherche de relations causales intégrant le rôle des phénomènes climatiques (le temps) et les caractères topo-structuraux du transect étudié (l'espace).

\section{LES MODALITES DE L'ETUDE}

\subsection{Le climat}

Le tronçon de route surveillé comprend près de $4 \mathrm{~km}$ en amont de la confluence de la Vésubie avec le Var, soit entre Plan du Var et le Cros d'Utelle (fig. 1). D'aval en amont, la vallée dont l'altitude est d'environ

\footnotetext{
* Laboratoire de Géographie, Université de Nice U. E. R. Lettres et Sciences humaines 98, Bd. E. Herriot, 06036 Nice Cedex.
} 


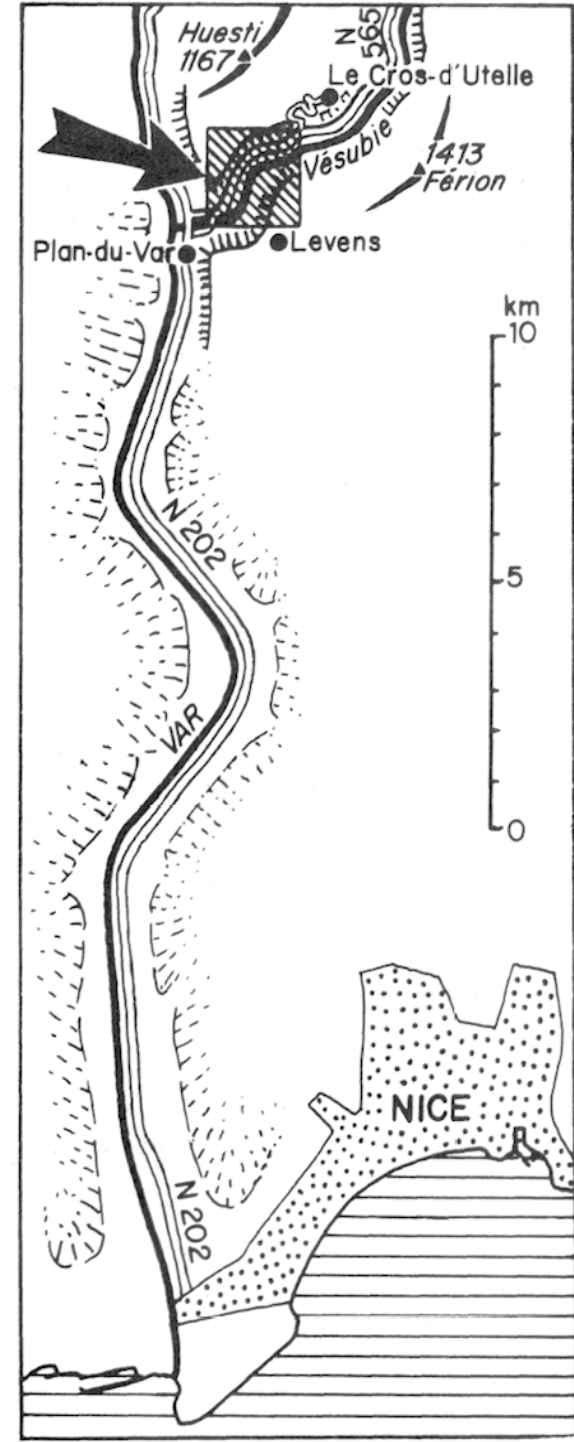

Fig. 1. CROQUIS DE SITUATION

$200 \mathrm{~m}$ présente d'abord un aspect de gorges escarpées avec de forts abrupts taillés dans les calcaires et marno-calcaires du Jurassique supérieur. A mi-parcours, la vallée s'élargit sensiblement et les versants sont tapissés à la base par des lambeaux de terrasses recouverts par des éboulis quaternaires à matrice sableuse et cailloutis ou même par des éboulements anciens incluant des blocs de taille parfois supérieure à 1 mètre. La route mord dans ces formations superficielles pratiquement sans végétation et s'enfonce dans de nouvelles gorges localement parsemées de quelques chênes verts, moins prononcées que les précédentes qui affectent cette fois les couches marno-calcaires du Crétacé inférieur.

D'un point de vue climatique, deux éléments - mesurés quotidiennement sont essentiels pour l'étude : les précipitations et les températures. Le régime méditerranéen des pluies est de rigueur avec une forte variabilité (maximum en automne), ainsi qu'une fréquence relativement élevée de pluies abondantes et/ou intenses. Exemple : $202 \mathrm{~mm}$ en 24 heures en octobre 1979, $50 \mathrm{~mm}$ en 30 minutes en septembre 1981 au Cros d'Utelle. Le régime thermique de cette vallée est original par rapport à celui de sa voisine : la brise nocturne forte dans la vallée du Var est ici quasi nulle. Cette absence de brassage de l'air facilite l'accumulation d'air très froid en hiver (85\% des températures minimales sont négatives durant les trois mois d'hiver) d'autant plus que certains secteurs du fond de la vallée masqués par le relief ne bénéficient d'aucune insolation au cours de cette période.

Au total, l'hypothèse de départ est que cette basse vallée montagnarde est soumise à deux agressions climatiques majeures engendrant une érosion :

- al ternances gel-dégel hivernales, et surtout

- saturation en eau des sols avec fort ruissellement liés à l'abondance épisodique des pluies.

\subsection{La méthodologie}

De décembre 1980 à septembre 1981,171 observations quotidiennes de l'état de la route ont été dffectuées (avant le déblaiement éventuel par le service des Ponts et Chaussées). A chaque observation ont été relevés le lieu exact des chutes de blocs éventuelles, ainsi que la masse éboulée en $\mathrm{kg}$ et la taille maximum des blocs en $\mathrm{cm}$.

La précision est évidemment a priori limitée, mais avec de l'entrainement les résultats de l'estimation sont très encourageants car proches de la réalité. De toutes façons il ne pouvait être question de peser chaque bloc (!), et plus que la valeur absolue du poids éboulé, l'important en est la valeur relative en fonction du temps et de l'espace.

Les résultats ont été soumis à divers traitements statistiques, et, alliés à une étude minutieuse des caractères topographiques et géologiques du versant bordant la route, ils ont permis de dégager treize secteurs le long de ce transect (fig. 2).

\section{LOCALISATION ET FREQUENCE DES CHUTES}

\subsection{Les lois de distribution des éboulements sur le transect entier}

Sur 171 observations, 57 jours de chutes ont été dénombrés, c'est-à-dire qu'un jour sur trois en moyenne un éboulement même minime s'est produit en un ou plusieurs secteurs du transect (fig. 3 ). 


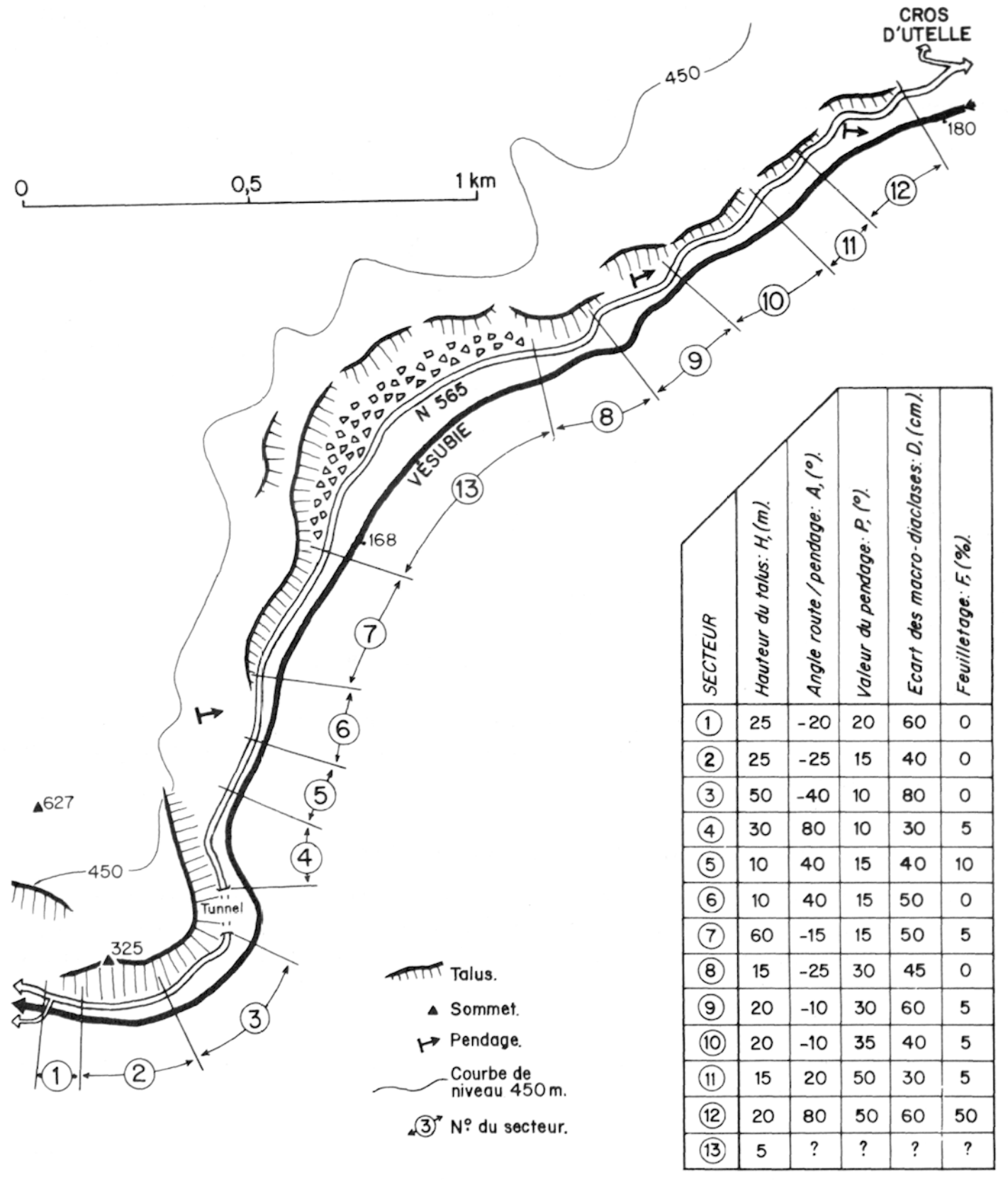

Fig. 2 : LIMITES DES SECTEURS ET VARIABLES TOPO - STRUCTURALES.

Au total, la masse éboulée durant ces six mois est estimée à plus de 3 tonnes (soit 1 tonne/km) réparties comme suit : dans la moitié des cas, le poids total quotidien est au plus égal à $5 \mathrm{~kg}$ et il ne dépasse $40 \mathrm{~kg}$ que dans $15 \%$ des cas (fig. 4 ).

Bien entendu, la distribution de ces chutes est irrégulière dans le temps comme le prouve le tableau I.

\begin{tabular}{|l|c|c|c|c|c|c|c|c|c|}
\hline & Déc. 80 & Jan. 81 & Fev. & Mars & Avril & Mai & Juin & Août & Sept. \\
\hline $\begin{array}{l}\text { Fréquence de } \\
\text { Jours de chutes }\end{array}$ & $54 \%$ & $75 \%$ & $37 \%$ & $29 \%$ & $24 \%$ & $27 \%$ & $5 \%$ & $0 \%$ & $31 \%$ \\
\hline Poids éboulé & $152 \mathrm{~kg}$ & $290 \mathrm{~kg}$ & $85 \mathrm{~kg}$ & $302 \mathrm{~kg}$ & $300 \mathrm{~kg}$ & $343 \mathrm{~kg}$ & $12 \mathrm{~kg}$ & $0 \mathrm{~kg}$ & $1234 \mathrm{~kg}$ \\
\hline
\end{tabular}




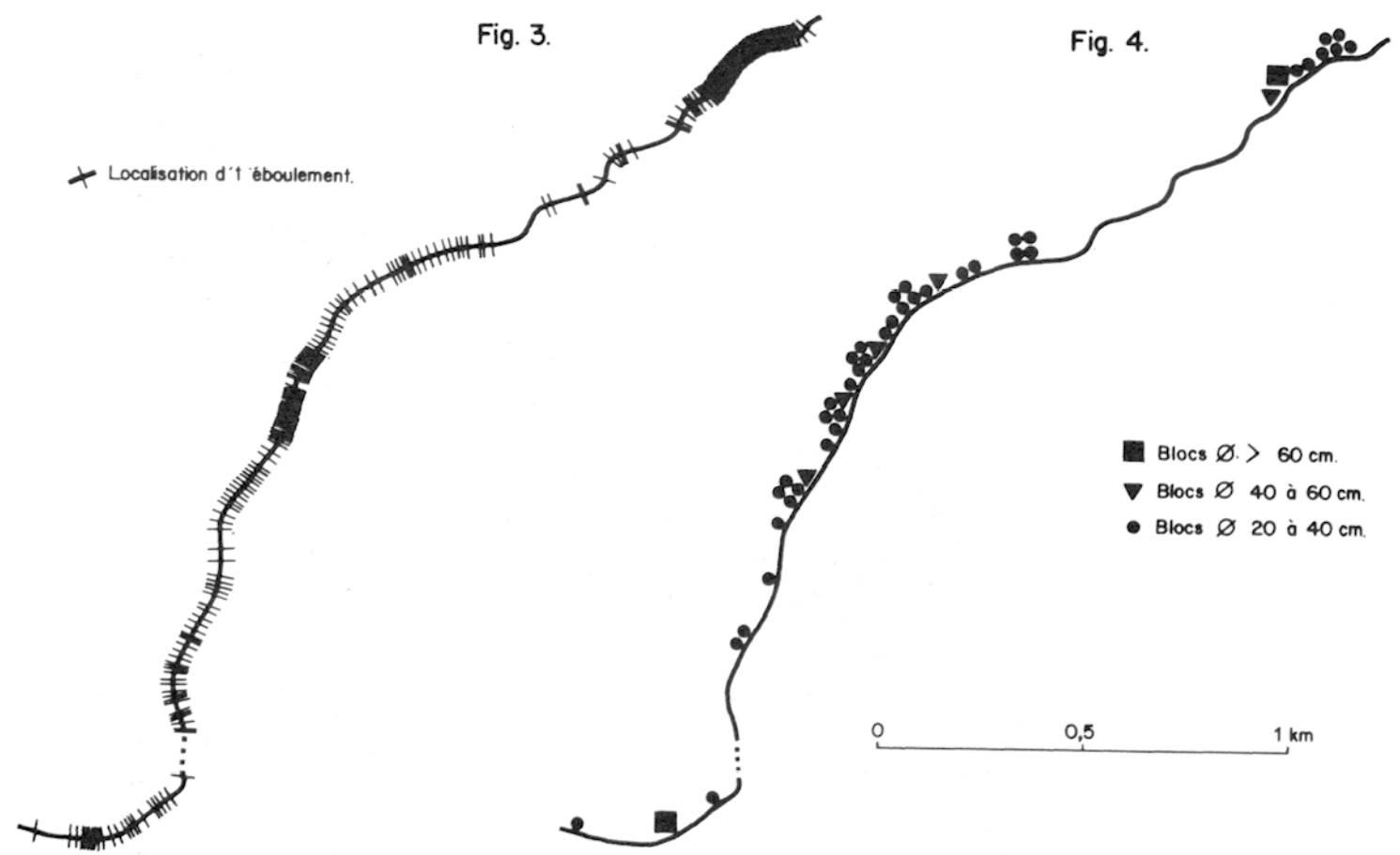

Fig. 3. LOCALISATION DES EBOULEMENTS (toutes chutes confondues).

Fig. 4. LOCALISATION DES GROS EBOULEMENTS.

Les éboulements sont nettement plus fréquents en hiver mais leur intensité est supérieure au printemps et en automne. Les neuf cas de blocs éboulés de taille supérieure à $40 \mathrm{~cm}$ se sont d'ailleurs tous produits en période de pluie non hivernale.

\subsection{La contribution de chaque secteur à la masse totale éboulée}

La longueur de chacun des secteurs varie de 100 à $350 \mathrm{~m}$ pour la roche en place, et atteint $650 \mathrm{~m}$ pour le secteur 13, celui de l'éboulis quaternaire. Au total, il apparaît que certains secteurs sont peu dangereux, en particulier $1,8,9,10$, tandis que d'autres sont exposés à de nombreux et parfois abondants éboulements : $5,7,11,12,13$.

\begin{tabular}{|c|c|c|c|c|c|c|c|c|c|c|c|c|c|}
\hline Secteur & 1 & 2 & 3 & 4 & 5 & 6 & 7 & 8 & 9 & 10 & 11 & 12 & 13 \\
\hline Longueur en $\mathrm{m}$ & 200 & 300 & 160 & 160 & 100 & 150 & 360 & 150 & 150 & 230 & 100 & 350 & 650 \\
\hline Fréq. de chutes & $2 \%$ & $23 \%$ & $23 \%$ & $39 \%$ & $30 \%$ & $12 \%$ & $25 \%$ & $5 \%$ & $7 \%$ & $14 \%$ & $30 \%$ & $54 \%$ & $68 \%$ \\
\hline $\begin{array}{l}\text { Taux d'éboule- } \\
\text { ment pour }\end{array}$ & $5 \mathrm{~kg}$ & 58 & 20 & 84 & 144 & 22 & 108 & 2 & 5 & 7 & 186 & 202 & 147 \\
\hline $\begin{array}{l}\text { T. E. par } \\
\text { chute }\end{array}$ & $5 \mathrm{~kg}$ & 4,5 & 1,5 & 3,8 & 8,5 & 3,1 & 7,7 & 0,7 & 1,2 & 0,9 & 10,9 & 6,5 & 3,8 \\
\hline
\end{tabular}

TABLEAU $\|$ - CHUTES PAR SECTEURS

Les taux moyens d'éboulement pour $100 \mathrm{~m}$ par jour de chute sont maximum dans certains secteurs marno-calcaires ( 5 et 11 surtout) : dans le secteur 11 les chutes sont deux fois moins fréquentes qu'en secteur 13 (ancien éboulis) mais quand elles se produisent, les quantités sont trois fois plus grandes.

\section{3 : Le rôle des saisons dans les éboulements}

Une analyse plus fine montre que pour un mois donné, chaque secteur est affecté par les éboulements de manière différentielle. 


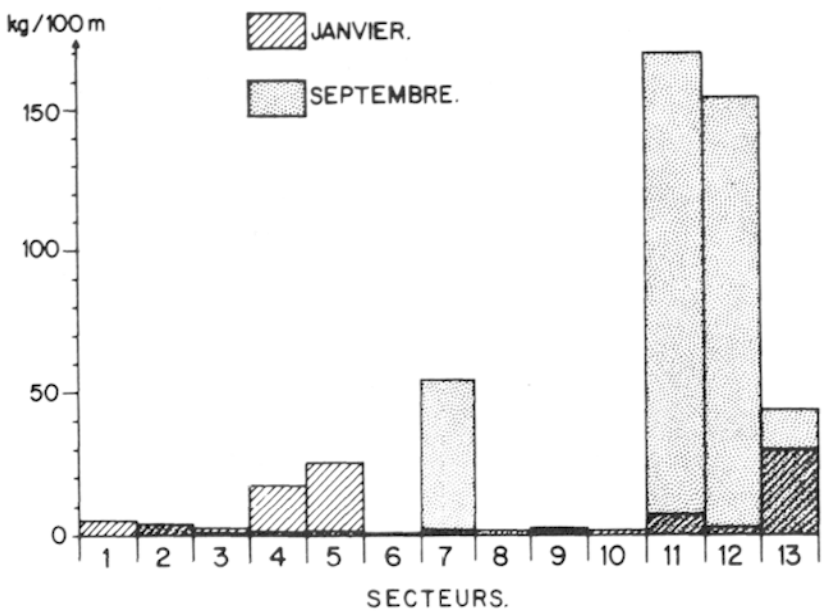

Fig. 5 : TAUX D'EBOULEMENT/100 m PAR SECTEUR, EN JANVIER ET SEPTEMBRE.
Les chutes hivernales sont surtout le fait du secteur $13: 59 \%$ du total de tous les secteurs en décembre, $65 \%$ en janvier, $61 \%$ en février. Sur cette matrice sableuse liant les cailloutis, les alternances gel-dégel ont ici le plus d'effet (fig. 5).

Avec la saison des pluies par contre, la situation s'inverse : ce sont alors certains secteurs marno-calcaires qui sont les plus érodés, avec un taux de $170 \mathrm{~kg} / 100 \mathrm{~m}$ en septembre dans le secteur 11 contre 44 dans le secteur 13 .

Il est donc clair que le rôle des saisons suit une logique différente selon les secteurs. Les chutes hivernales fournissent $1 / 3$ de son total au secteur 13 mais seulement 3 à $5 \%$ aux secteurs 11 ou 12 .

\section{LES LOIS DE CAUSALITE. ESSAI DE MODELISATION}

\subsection{Limites de l'analyse univariée}

Empiriquement, il suffit de confronter les chutes de pierres avec les températures minimales et les précipitations mesurées quotidiennement au Cros d'Utelle pour confirmer l'hypothèse de départ : il y a des relations évidentes entre elles :

\begin{tabular}{|c|c|c|c|c|c|c|c|c|}
\hline & Déc. 80 & Jan. 81 & Fév. & Mars & Avril & Mai & Juin & Sept. \\
\hline Chutes sans pluie ni gel & - & - & $12 \%$ & $9 \%$ & - & - & - & - \\
\hline Gel sans chutes & - & $13 \%$ & $6 \%$ & $26 \%$ & - & - & - & - \\
\hline Pluie sans chutes & - & - & - & - & $19 \%$ & $18 \%$ & - & - \\
\hline $\begin{array}{l}\text { Moyenne des } t^{\circ} \\
\text { minimales }\end{array}$ & $-3,8$ & $-4,2$ & 0,5 & $-0,6$ & 3,5 & 7,8 & 15,7 & 14,4 \\
\hline $\mathrm{T}^{\circ}$ minimale absolue & $-5,8$ & $-8,4$ & 3,9 & $-3,5$ & 0,7 & 5,8 & 15,7 & 10,5 \\
\hline Fréquence des gelées & $100 \%$ & $93 \%$ & $66 \%$ & $62 \%$ & - & - & - & - \\
\hline Total des pluies en mm & 0 & 4,1 & 12,4 & 90,5 & 23,9 & 51,6 & - & 115,5 \\
\hline $\begin{array}{l}\text { Pluie maximale en } 24 \mathrm{~h} \\
\text { en } \mathrm{mm}\end{array}$ & 0 & 4,1 & 7,4 & 41,9 & 12,2 & 29,7 & & 51,2 \\
\hline
\end{tabular}

TABLEAU III: CHUTES ET PHENOMENES CLIMATIQUES RELEVES LORS DE CES CHUTES

La faible fréquence des chutes non expliquées ou des situations favorables non suivies de chutes prouve cette forte dépendance qu'une fois constatée il est encore plus intéressant de quantifier à l'aide d'analyses multivariées. la régression.

Parmi les techniques de l'analyse de données, l'algorithme capable de répondre à cette question est

\subsection{Le rôle des éléments climatiques}

Outre le froid et la pluie, un troisième facteur peut être responsable d'éboulements : le vent sec (vent de NW avec effet de foëhn). Son efficacité paraît accrue lorsqu'il fait suite à la pluie et pourrait être liée à une rétraction du matériau par brutale déshydratation. Faute de mesures cette variable "vent seç» n’a pas été retenue.

Par contre, pour tester le rôle des facteurs climatiques sur le poids des blocs éboulés $Y$, trois variables 
sont retenues : les températures minimales négatives, $\mathrm{xl}$; les précipitations tombées dans les $24 \mathrm{~h}$ précédant la chute, $x 2$; les pluies à $-72 \mathrm{~h}, \mathrm{x} 3$. Cette dernière variable intègre partiellement les phénomènes à forte inertie (imbition du sol en eau), et les effets de synergie (la gélifraction est plus forte après la pluie, dans un matériau détrempé).

Dans le secteur 13 (éboulis) pour l'ensemble des chutes quotidiennes, x1 et x3 sont peu liées à $Y$, le coefficient de corrélation $R$ n'étant que de 0,29 pour chacun. Par contre $x 2$ et $Y$ ont un $R$ de 0,72 .

Une régression multiple prenant simultanément en compte les trois variables donne un $\mathbf{R}$ de 0,74 . La standardisation des coefficients de régression indique que la quantité de pierres éboulées s'explique avant tout par la pluie récente $(-24 \mathrm{~h})$, suivie de loin par le froid et accessoirement par la pluie plus ancienne $(-72 \mathrm{~h})$.

En d'autres secteurs - en roche mère marno-calcaire cette fois - $\mathrm{x} 2$ est encore la variable la plus corrélée avec $Y$, mais en diverses proportions : $R=0,77$ pour le secteur $7 ; 0,55$ seulement pour les secteurs 9 , $10,11,12$.

Le gel, sans grand rôle, cède cette fois la place de seconde variable par ordre d'importance aux pluies anciennes dont $R=0,42$ dans les secteurs 9 et 12 . Des matériaux différents ne réagissent donc pas de la même manière à une agression climatique donnée. Les coefficients de corrélation même multiples bien que significatifs ne sont pas très élevés, qu'ils s'appliquent à une régression linéaire comme ici ou à une fonction puissance comme l'ont montré des essais. Il est très possible qu'une série plus longue donne de meilleurs résultats, limités toutefois par la complexité du problème : si la variation de masse éboulée n'est pas totalement proportionnelle à la variation des éléments climatiques, c'est que deux "causes» climatiques identiques n'ont pas le même "effet» en terme de poids éboulé. En effet, bien qu'au même endroit, elles ne s'appliquent pas en réalité au même terrain, celui-ci ayant évolué durant le laps de temps qui sépare les deux «causes»: un bloc à la limite du déséquilibre s'éboulera à la première averse ; la même averse n'ayant plus d'effet quelque temps plus tard. A l'échelle de temps courte de la chute de blocs sous la pluie par gravité, il faut donc juxtaposer une échelle temporelle plus longue, celle de la préparation du matériel (élargissement des fissures par la décompression agissant sur les flancs des gorges), altération qui permettront le détachement de blocs.

En bref, il ressort cependant que si l'alternance gel-dégel foumit de nombreux débris rocheux de petite dimension (en secteur d'éboulis surtout), le type de temps le plus dangereux est une forte chute de pluie et ce, dans les heures qui accompagnent ou qui suivent cette précipitation.

\subsection{Le rôle de la topographie et de la structure (fig. 6)}

Les variables climatiques rendent donc en grande partie compte de la différenciation temporelle, mais ne peuvent être à l'origine des variations spatiales constatées, car la portion de vallée étudiée est de dimensions trop réduites pour ce faire. En toute hypothèse, ce sont donc des variables géomorphologiques qui rendent compte de l'inégale répartition spatiale des éboulements.

$\mathrm{Au}$ total, cinq variables explicatives sont retenues (fig. 2). La première, topographique, est la hauteur en mètres de la partie verticale ou subverticale $\left(>80^{\circ}\right)$ du talus bordant la route côté amont, $x 1$. A priori on peut penser que le risque d'éboulement augmente avec la valeur angulaire de la pente du talus. D'ailleurs, les parois calcaires verticales sont parsemées de taches beiges détonnant sur le gris lié à l'oxydation (manganèse), et qui sont les cicatrices du départ des blocs aspirés par l'appel au vide. Ce dernier n'est qu'une potentialité suivie d'effet si la structure fournit du matériel à l'énergie de gravité. La cohésion de la roche est, dans cette optique, essentielle : forte dans les calcaires durs, massifs et peu fissurés, elle est faible dans l'éboulis à matrice sableuse sauf là où localement les eaux riches en carbonates ont cimenté le matériel en brèches.

Cette cohésion est très difficile à quantifier pour des roches de texture aussi opposées qu'éboulis et calcaire plus ou moins marneux. On peut l'approcher par le biais de variables structurales, ce qui exclut malheureusement l'éboulis quaternaire du modèle.

$\mathrm{x} 2$ est l'angle fait par l'orientation du pendage avec celle de la route vue en plan ; x3 est la valeur angulaire du pendage (par rapport à l'horizontale) : on conçoit aisément qu'un fort pendage dirigé vers la route soit favorable à des détachements de blocs, suivis de leur chute. Ce détachement est facilité dans les zones de faiblesses, fortement fissurées par des macro-diaclases.

L'écartement moyen entre celles-ci (en $\mathrm{cm}$ ) constitue la variable $\mathrm{x} 4$. Quant à $\mathrm{x} 5$, elle concerne le pourcentage de structure feuilletée affectant localement les marno-calcaires qui se débitent aisément en minces feuillets friables lorsque $\times 5$ est fort. 

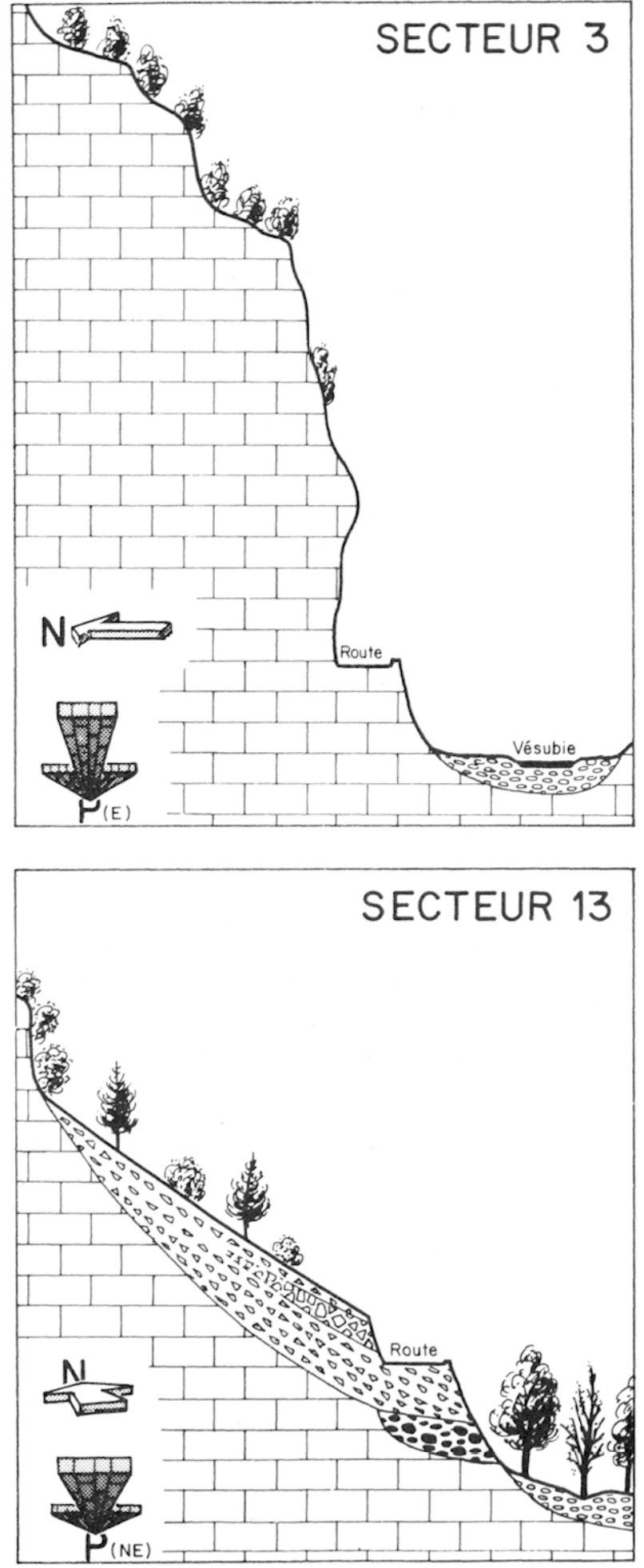

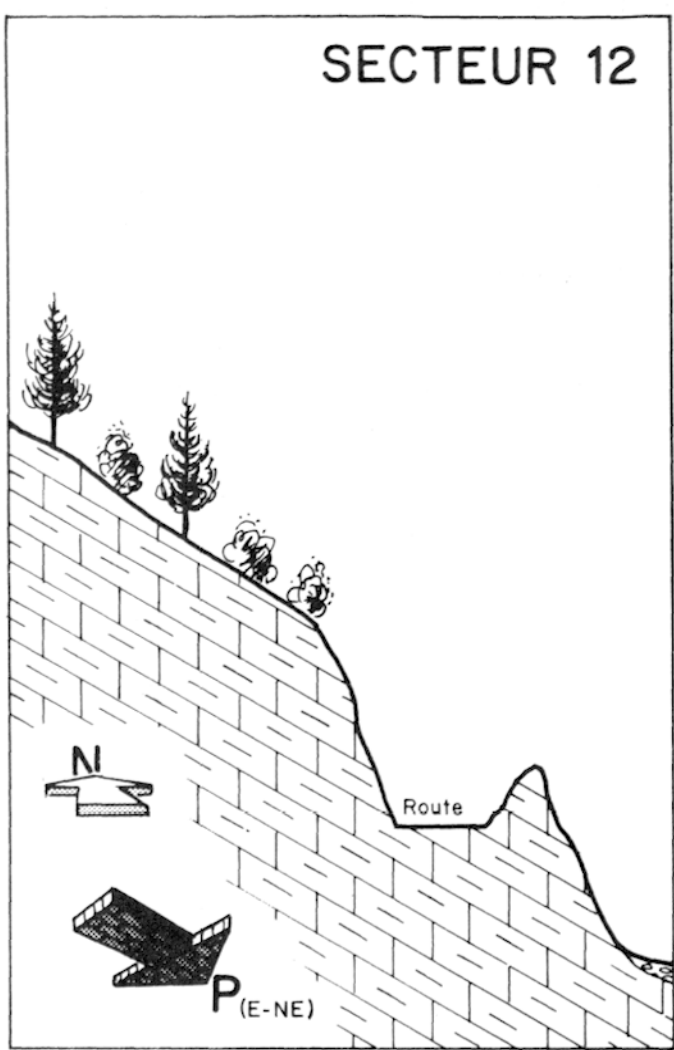

0 10 $20 \mathrm{~m}$

\section{STRUCTURE:}

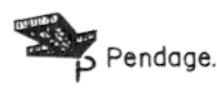

8:89 Alluvions actuelles.

एo. Eboulis ancien lité.

एव: Eboulement ancien.

5-8. Terrasse quaternaire.

I, Calcaire.

ND Marno-calcaire

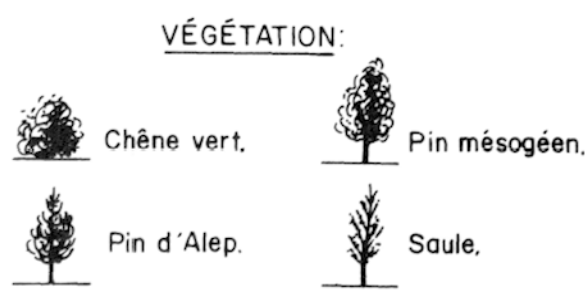

Fig. 6 : COUPE DES SECTEURS 3, 12, 13.

Ces variables ont été relevées avec soin tout au long du tronçon routier étudié et leur sélection paraît encourageante :

L'équation $Y=174+4,9 \times 1-0,59 \times 2-1,1 \times 3-4,7 \times 4+16 \times 5$ est un modèle performant puisque $\mathrm{R}=0.95$. Il ajuste le poids total des éboulements pour chacun des 12 secteurs (le treizième étant exclu).

Les variables les plus déterminantes sont dans l'ordre : le feuilletage, puis la hauteur du talus subvertical, et la densité des diaclases. Le même modèle appliqué cette fois à des dimensions comparables : les taux de chute $/ 100 \mathrm{~m}$ concernant comme ci-dessus toutes les chutes donne de moins bons résultats car $R=0.83$, mais le poids respectif des variables dans la régression reste inchangé. 


\section{4. Le poids des variables topo-structurales en fonction des variables climatiques}

Si l'on effectue une régression sur les taux de chute mensuels des variations apparaissent :

En janvier $R$ est nettement moins élevé $(0.71)$ qu'en septembre $(0.90)$, et le «poids» des variables évolue. Le feuilletage par exemple, globalement important s'efface complètement dans ces régressions d'hiver et d'automne ; il est efficace sous les faibles pluies dans la mesure où celles-ci ne peuvent mobiliser qu'un matériel peu abondant.

Le pendage, en moyenne sans grand effet, est au contraire déterminant sous les fortes pluies d'automne d'autant plus lorsqu'il est orienté vers la route et de forte inclinaison, comme le prouve le modèle pour les secteurs $12,4,5,6$.

La densité des fissures proportionnellement sans grand effet sous les fortes pluies de septembre, a au contraire une grande importance en janvier ; ce qui suggère que ces fissures remplies d'eau transformée en glace l'hiver offrent un bon levier d'action à l'érosion.

Enfin, la hauteur du talus subvertical est plus déterminante pendant la saison pluvieuse qu'en hiver, ce qui semble indiquer qu'une importante surface de départ ces blocs (grande hauteur du talus) offre davantage de prises à l'érosion pluviale qu'au gel.

\section{CONCLUSION}

Le fait que les secteurs à haut risque ne soient pas exactement les mêmes selon les saisons s'explique par le fait que les variables explicatives géomorphologiques pèsent d'un poids différent sur les régressions selon le type de temps : gel intense, faibles précipitations, averses violentes, etc...

Pour être réellement prédictif un modèle de ce type doit pouvoir être bâti sur une longue série de mesures intégrant tous les types de temps et gagnerait à se fonder sur un espace plus étendu. Son efficacité peut être améliorée par une réflexion supplémentaire sur la nature et la mesure des variables considérées comme explicatives de $\mathrm{Y}$ : étude poussée de la résistance mécanique, de la macro et micro-fissuration ... Cependant une amélioration sensible des résultats passe probablement par une sophistication nettement accrue des moyens, jusqu'ici très réduits.

Il nous paraît en tout cas certain que comme le montre cette étude, la solution des problèmes posés par l'érosion exige une prise en compte de tous les éléments du milieu à la fois. Tous ces éléments étant en interaction, une vision globale des phénomènes s'impose sous peine d'échec.

\section{BIBLIOGRAPHIE}

CARREGA P.,(1980). - Note sur le gradient thermique nocturne hivernal des basses vallées du Var et de la Vésubie. Bull. de la Commission Météorologique des Alpes Maritimes. pp. 32-36.

CARREGA P.,(1982). - Les facteurs climatiques limitants dans le Sud des Alpes Occidentales, Thèse 3ème Cycle, Nice. $251 \mathrm{p}$..., (ronéo).

GOGUEL J.,(1980).-Géologie de l'environnement, Masson.

JULIAN M., (1976).- Les Alpes-Maritimes franco-italiennes - Etude géomorphologique, Thèse lettres Aix enProvence. Imp. Univ. Lille III.

LETOURNEUR J. et MICHEL R.,(1971)-Géologie du Génie civil, A. Colin. 724 p.

NICOD J., (1975).- Sur l'évolution des versants des canyons karstiques dans les régions méditerranéennes. Actes $d u$ Symposium sur les versants en pays méditerranéen. Aix-en-Provence, pp 15-20.

TRICART J.,(1979)-_Géomorphologie applicable, Masson.

TRICART J. et KILIAN J.,(1979)-L'écogéographie et l'aménagement du milieu naturel, Maspéro, 326.

VOIGHT B.,(1978),-Rockslides and Avalanches, 1 - Natural phenomenes, Elsevier. 823 p. 